\title{
SELECCIÓN DE MAÍCES CRIOLLOS PARA SU APLICACIÓN EN LA INDUSTRIA CON BASE EN SU VALOR AGREGADO
}

\section{MAIZE LANDRACES SELECTION FOR INDUSTRIAL END-USE BASED ON THEIR ADDED VALUE}

\author{
Marcela Gaytán-Martínez ${ }^{1,2 \star}$, Juan de D. Figueroa-Cárdenas ${ }^{1}$, María de la L. Reyes-Vega ${ }^{3}$, \\ Eduardo Morales-Sánchez ${ }^{4}$ y Froylán Rincón-Sánchez ${ }^{5}$
}

\begin{abstract}
${ }^{1}$ Centro de Investigación y de Estudios Avanzados, Instituto Politécnico Nacional (IPN). Libramiento Norponiente 2000. 76230, Fracc. Real de Juriquilla, Querétaro, Qro. ${ }^{2}$ Programa de Posgrado en Alimentos del Centro de la República, Research and Graduate Studies in Food Science, School of Chemistry, Universidad Autónoma de Querétaro. Cerro de las Campanas s/n. 76000, Col. Centro. Querétaro, Qro. ${ }^{3}$ Food Research Department, Universidad Autónoma de Coahuila, Blvd. V. Carranza s/n. 25280, Saltillo, Coahuila. ${ }^{4}$ Centro de Investigación en Ciencia Aplicada y Tecnología Avanzada del IPN, Unidad Querétaro. Cerro Blanco No. 141. 76090, Col. Colinas del Cimatario, Santiago de Querétaro, Querétaro. ${ }^{5}$ Universidad Autónoma Agraria Antonio Narro. Calz. Antonio Narro 1923. 25070, Buenavista, Saltillo, Coahuila.
\end{abstract}

*Autor para correspondencia (marcelagaytanm@yahoo.com.mx)

\section{RESUMEN}

En los últimos años se ha incrementado el estudio, mejoramiento y cultivo de maíces nativos (Zea mays L.), con la finalidad de orientarlos hacia un uso final específico y así aumentar su valor en beneficio de los productores. El presente trabajo tuvo como objetivo determinar el posible uso final de seis maíces criollos de razas diferentes (Bolita, Elotes Cónicos, Cónico Norteño, Pepitilla, Pinto y Zapalote), de acuerdo con sus características físicas, químicas, térmicas y de calidad nixtamalera. Las características físicas determinadas fueron: peso de cien granos, dureza, índice de flotación, cristalinidad y tamaño de gránulo de almidón, porcentaje de endospermo, pericarpio y germen; las químicas: proteínas, cenizas y lípidos; las térmicas: entalpía temperatura de gelatinización y viscosidad relativa; y las de calidad nixtamalera: rendimiento y textura para masa y tortilla. Se aplicó un análisis por componentes principales para definir cuáles maíces tienen un uso final específico en la industria. Con esta metodología se encontró que la accesión de la raza Bolita cumplió con las características necesarias para ser destinada a la industria de las botanas, la accesión de la raza Cónico Norteño a la industria de las harinas instantáneas; las accesiones de las razas Cónico Norteño, Zapolote y Pepitilla a la industria de la masa y la tortilla; las accesiones de las razas Pinto y Elotes Cónicos a la industria de la molienda seca..

Palabras clave: Zea mays, maíces nativos, nixtamalización, uso final, maíces de alto valor agregado.

\section{SUMMARY}

In recent years, the study, improvement and cultivation of maize landraces (Zea mays L.) have increased with the purpose of enhancing value to benefit producers and increase end uses. This study classifies kernels from six accessions of different maize landraces (Bolita, Elotes Cónicos, Cónico Norteño, Pepitilla, Pinto and Zapalote) based on physical, chemical, thermal and nixtamal quality properties for end usage. Physical properties measured were 100-kernel weight, hardness, flotation index, crystalinity, starch granule size and percentage of endosperm, germ, and pericarp. Chemical properties tested included proteins, lipids and ashes. Gelatinization temperature, enthalpy and relative viscosity were tested within the thermal properties group. For nixtamal quality, yield and texture for masa and tortillas was assesed. A principal component analysis was applied to identify specific end use in the industry for each accession. Based on this methodology, the
Bolita landrace accession has appropriate characteristics for the snack industry; the Cónico Norteño landrace accession is adequate for the instant flour industry. Accessions from landraces Zapolote, Pepitilla and Cónico Norteño, has suitable characteristics for the masa and tortilla industry. Finally, accessions from landraces Pinto and Elotes Cónicos can satisfactorily be used for the wet milling industry.

Index words: Zea mays, maize landraces, nixtamalization, end-use, value enhanced maize.

\section{INTRODUCCIÓN}

El término "maíz criollo" se utiliza para denotar variedades de maíz (Zea mays L.) nativas a una comunidad, región o estado, y tales variedades están conformadas por poblaciones heterogéneas de plantas, las cuales son diferenciadas por los agricultores por su color, textura, forma del grano, forma de la mazorca, ciclo de cultivo y uso. Estos materiales genéticos han sido desarrollados por los agricultores a través de múltiples ciclos de selección empírica, y los conservan y manejan bajo un esquema de agricultura tradicional con un complejo sistema de intercambio de semillas (Aragón et al., 2006).

La siembra del maíz nativo (MN) está disminuyendo, debido, entre otros factores, a que no encuentran un nicho de mercado apropiado, por lo que el precio que se paga es castigado (Trujillo, 2003). Esto es preocupante porque de seguir así, se estima que en poco tiempo los MN podrían perder el carácter de maíces especializados y no tendrían características propias que los distingan de los demás maíces, lo que causaría una importante pérdida de diversidad genética.

Por ello es importante que, además de evaluar los aspectos botánicos, genéticos, agronómicos, citológicos o descriptores fenotípicos de la mazorca o grano (Wellhausen et al., 1952), se estudien las características físicas, químicas, 
térmicas y de calidad nixtamalera, así como su relación con el uso final (Narváez et al., 2007a). Tales estudios permitirían que los maíces sean identificados como material de mayor valor agregado; es decir, que posean las características que la industria requiere para la producción de una diversa gama de productos y subproductos, de modo que la industria pagaría un sobreprecio por la especificidad del producto (Serna, 1999; US Grain Council, 2001). El objetivo del presente trabajo fue determinar el uso final más apropiado de seis poblaciones nativas de maíz, con base en sus características físicas, químicas, térmicas y de calidad nixtamalera, para dar valor agregado a los MN.

\section{MATERIALES Y MÉTODOS}

El material genético analizado estuvo conformado por seis accesiones de maíces nativos provenientes del Programa de Recursos Fitogenéticos de la Universidad Autónoma Agraria "Antonio Narro" (UAAAN). Los materiales genéticos y su clasificación racial (en paréntesis) fueron los siguientes: Compuesto Elotes Cónicos (Elotes Cónicos), Compuesto Bolita (Bolita), Compuesto Maíz Pinto en lo sucesivo nombrado como Pinto (Sin clasificación racial), Jagüey (Cónico Norteño), Zapalote (Zapalote) y Mor149 (Pepitilla). La semilla de los materiales fue incrementada a través de polinizaciones controladas a mano en el ciclo agrícola Otoño-Invierno 2003, en un campo experimental ubicado en Tepalcingo, Mor. ( $18^{\circ} 36^{\prime} \mathrm{LN}$ y $98^{\circ} 50^{\prime} \mathrm{LO}$, altitud de $1182 \mathrm{~m}$ ), con precipitación media anual de $877 \mathrm{~mm}$ y un clima cálido subhúmedo (Awo).

\section{Propiedades físicas y químicas de los maíces}

Las características fisicoquímicas medidas fueron: peso de 100 granos (PCG); índice de flotación (IF) y dureza. La composición anatómica se determinó por disección manual de 10 granos de cada tipo de maíz en los que se calcularon los porcentajes de endospermo (E), pericarpio $(P)$ y germen $(G)$. Se determinó el tamaño de gránulo de almidón (TGA) con un microscopio electrónico de barrido. Estas mediciones se hicieron de acuerdo con los métodos reportados por Narváez-Gónzalez et al. (2006) y Narváez et al. (2007a). La cristalinidad de los maíces fue evaluada mediante el área bajo la curva de los difractogramas obtenidos por medio de espectroscopía de difracción de rayos-X (Wang et al., 2006). Los contenidos de proteína (método 954), lípidos (método 920.39) y cenizas (método 923.03) se hicieron conforme a los métodos de la AOAC (2002).

\section{Propiedades térmicas}

Se midió la temperatura de gelatinización y la entalpía con un equipo CBD (Mettler Toledo $821 \AA$, Schwerzenbach, Suiza). La viscosidad relativa se determinó con un aparato denominado Rapid Visco Analyser 3C® (Newport Scientific PTY LTD, Sidney, Australia). Estas técnicas fueron descritas por (Narváez-González et al. (2006) y por Narváez et al. (2007 b).

\section{Calidad nixtamalera}

La nixtamalización de las seis accesiones de maíces criollos se llevó a cabo de acuerdo con lo descrito por Salinas y Vázquez (2006). Las masa obtenidas de los maíces criollos nixtamalizados se deshidrataron en un secador tipo flash (Fabricado por CINVESTAV-Unidad Querétaro, Marca propia) a $270 \pm 5{ }^{\circ} \mathrm{C}$, con un tiempo de residencia de 10 s. De cada harina se pesó 500 g y se agregó el agua necesaria para formar una masa. Se midió el rendimiento (RM) y la textura de la masa (cohesión y la adhesión), según el método descrito por Mauricio et al. (2004). Las masas se troquelaron para obtener las tortillas y en ellas se evaluó: rendimiento (RT), pérdida de peso (PP) y textura de las tortillas (fuerza al corte y a la tensión), de acuerdo con lo reportado Mauricio et al. (2004).

\section{Análisis estadístico}

Se utilizó un diseño completamente aleatorio. Además del análisis de varianza y la comparación de medias por Tukey, se calculó la correlación entre las variables evaluadas por medio de la correlación de Pearson. Se efectuó un análisis de componentes principales (SAS Institute, 2004) y la clasificación de muestras se hizo de acuerdo con lo reportado por Mauricio et al. (2004).

\section{RESULTADOS Y DISCUSIÓN}

\section{Propiedades físicas y químicas}

$\mathrm{El}$ análisis de varianza mostró diferencias significativas $(\mathrm{P}<0.05)$ entre variedades en cuanto a las variables físicas y químicas del grano (Cuadro 1). El maíz Pepitilla presentó el menor peso de 100 granos (PCG) con $16 \mathrm{~g}$, en tanto que Elotes Cónicos tuvo el mayor (PCG)con 36.2 g. Los maíces menos densos fueron Pinto, Elotes Cónicos, Pepitilla y Zapalote (con 93, 91, 85 y $82 \%$, respectivamente) y el más denso fue Bolita (23\%). Salinas et al. (1992) reportaron que el índice de flotación (IF) se usa para medir de manera indirecta la dureza del grano; esto corresponde con los datos encontrados en este estudio, ya que la dureza presentó una correlación significativa con el IF $(r=-0.630, \mathrm{P}<0.05)$.

Los almidones contienen estructuras cristalinas y no cristalinas; la relación entre éstas es el principal factor que determina las propiedades del almidón. La combinación de estas estructuras da como resultado un patrón típico de difracción de rayos X, por lo que dicho patrón depende del 
Cuadro 1. Medias de las propiedades físicas y químicas de los maíces nativos.

\begin{tabular}{|c|c|c|c|c|c|c|c|c|c|c|c|}
\hline Maíz nativo & $\begin{array}{l}\mathrm{PCG}^{\dagger} \\
(\mathrm{g})\end{array}$ & $\begin{array}{l}\mathrm{IF}^{\dagger \dagger} \\
(\%)\end{array}$ & $\begin{array}{r}\text { Dureza } \\
(\mathrm{kg}-\mathrm{F})\end{array}$ & $\begin{array}{l}\mathrm{TGA}^{\xi} \\
(\mu \mathrm{m})\end{array}$ & $\begin{array}{l}\mathrm{C}^{\tau} \\
(\%)\end{array}$ & $\begin{array}{l}\mathrm{E}^{9} \\
(\%)\end{array}$ & $\begin{array}{l}\text { P9g } \\
\text { (\%) }\end{array}$ & $\begin{array}{l}\mathrm{G}^{\xi \xi} \\
(\%)\end{array}$ & $\begin{array}{l}\text { Proteínas } \\
\text { (\%) }\end{array}$ & $\begin{array}{c}\text { Lípidos } \\
(\%)\end{array}$ & $\begin{array}{c}\text { Cenizas } \\
(\%)\end{array}$ \\
\hline Elotes cónicos & $36.2 \mathrm{a}$ & $91 \mathrm{a}$ & $11.2 \mathrm{bc}$ & $11.68 \mathrm{ab}$ & $10.66 \mathrm{a}$ & $82.57 \mathrm{ab}$ & $4.55 \mathrm{~b}$ & $12.87 \mathrm{a}$ & $9.65 \mathrm{~d}$ & $4.3 \mathrm{a}$ & $1.47 \mathrm{a}$ \\
\hline Bolita & $32.1 \mathrm{a}$ & $23 c$ & $14.6 \mathrm{a}$ & $12.07 \mathrm{a}$ & $9.20 \mathrm{~b}$ & $83.96 \mathrm{a}$ & $4.16 \mathrm{~b}$ & $10.24 \mathrm{~b}$ & $10.22 \mathrm{~cd}$ & $4.81 \mathrm{a}$ & $1.50 \mathrm{a}$ \\
\hline Pinto & $33.4 \mathrm{a}$ & $93 \mathrm{a}$ & $11.3 \mathrm{bc}$ & $8.13 c$ & $11.13 \mathrm{a}$ & $82.52 \mathrm{ab}$ & $3.69 \mathrm{~b}$ & $11.95 \mathrm{ab}$ & $10.80 \mathrm{c}$ & $4.36 \mathrm{a}$ & $1.59 \mathrm{a}$ \\
\hline $\mathrm{CN}^{\dagger}$ & $20.7 \mathrm{bc}$ & $60 \mathrm{~b}$ & $9.3 \mathrm{~cd}$ & $12.40 \mathrm{a}$ & $9.44 \mathrm{~b}$ & $83.55 \mathrm{a}$ & $4.71 \mathrm{~b}$ & $10.24 \mathrm{~b}$ & $10.24 \mathrm{~cd}$ & $3.72 \mathrm{a}$ & $1.50 \mathrm{a}$ \\
\hline Zapalote & $22.3 b$ & $82 \mathrm{a}$ & $8.7 \mathrm{~d}$ & $9.36 \mathrm{bc}$ & $10.72 \mathrm{a}$ & $84.01 \mathrm{a}$ & $4.81 \mathrm{~b}$ & $10.65 \mathrm{~b}$ & $11.34 \mathrm{~b}$ & $3.74 \mathrm{a}$ & $1.58 \mathrm{a}$ \\
\hline Pepitilla & $16.0 \mathrm{c}$ & $85 \mathrm{a}$ & $11.8 \mathrm{~b}$ & $10.42 \mathrm{abc}$ & $10.76 \mathrm{a}$ & $80.84 \mathrm{~b}$ & $7.55 \mathrm{a}$ & $11.84 \mathrm{ab}$ & $11.93 \mathrm{a}$ & $4.34 \mathrm{a}$ & $1.06 \mathrm{~b}$ \\
\hline
\end{tabular}

Las medias con letras iguales en cada columna no son estadísticamente diferentes (Tukey, 0.05 ). $\mathrm{CN}^{\dagger}=$ Cónico Norteño; ${ }^{\mathrm{t}} \mathrm{C}=$ cristalinidad; ${ }^{\dagger} \mathrm{PCG}=$ peso de cien granos;

${ }^{\dagger \dagger} \mathrm{IF}=$ índice de flotación; ${ }^{\xi} \mathrm{TGA}=$ tamaño de gránulo de almidón; ${ }^{\circ} \mathrm{E}=$ endospermo; ${ }^{9} \mathrm{P}=$ pericarpio; ${ }^{\xi \xi} \mathrm{G}=$ germen.

tipo de almidón. Se ha demostrado que los almidones de cereales muestran un patrón de difracción tipo A, los de tubérculos producen un patrón tipo $\mathrm{B}$, y algunos tubérculos y granos dan un patrón tipo C (Wang et al., 2006). Las reflexiones de los difractogramas de los maíces en estudio presentaron los ángulos de Bragg 15, 17, 18.1 y $23^{\circ}$ en el ángulo $2 \theta$ (Figura 1), característicos de los almidones tipo A en cereales, cuya estructura presenta espacios de aproximadamente 5.8 a $3.84 \AA$ (Zobel, 1988).

El porcentaje de cristalinidad (Cuadro 1), obtenido del área bajo la curva de cada patrón de difracción de rayosX, presentó una amplitud de valores de $9.20 \%$ (Bolita) a $11.13 \%$ (Pinto). El porcentaje de cristalinidad correlacionó significativamente con IF $(r=0.820, P<0.01)$, tamaño del gránulo de almidón $(\mathrm{r}=-0.620, \mathrm{P}<0.05)$, viscosidad relativa $(r=0.900, P<0.001)$, y con el contenido de fibra $(0.786$, $\mathrm{P}<0.05)$. Los valores de tamaño de gránulo de almidón (TGA) del maíz Bolita (12.07 $\mu \mathrm{m})$, Cónico Norteño (12.40 $\mu \mathrm{m})$ y Pepitilla $(10.42 \mu \mathrm{m})$ no presentaron diferencias entre ellos, en tanto que Zapalote $(9.36 \mu \mathrm{m})$ y Pinto $(8.13 \mu \mathrm{m})$ no presentaron diferencias significativas en el porcentaje de cristalinidad. Según Narváez-González et al. (2006) y Narváez et al. (2007b), el TGA es un indicativo del grado de compactación del almidón de los endospermos duros y suaves. En el estudio que aquí se reporta, esta variable presentó correlaciones negativas significativas con IF $(\mathrm{r}=$ $-0.625, \mathrm{P}<0.05)$, contenido de proteína $(\mathrm{r}=-0.621, \mathrm{P}<0.05)$ $\mathrm{y}$ porcentaje de cristalinidad $(\mathrm{r}=-0.620, \mathrm{P}<0.05)$.

Se puede observar que las variables que correlacionan con la cristalinidad son un reflejo de la composición química del gránulo de almidón, es decir, de la relación de amilosa y amilopectina, así como del empaquetamiento dentro del gránulo que está relacionado con la dureza del grano (Narváez-González et al., 2006). Patindol y Wang (2003) reportaron datos similares para arroz (Oryza sativa L.), al encontrar una relación directa entre la cristalinidad y el contenido de amilopectina, y una relación inversa entre la cristalinidad y el contenido de amilosa. Al parecer, la inten- sidad de difracciones es determinada por volumen de amilopectina. Similarmente, Bochamikova et al. (2003) encontraron que la morfología del gránulo de almidón del trigo (Triticum aestivum L.) depende principalmente del arreglo de la amilopectina, y que no cambia significativamente con el arreglo de la amilosa.

El grano de maíz está constituido por pericarpio, germen y endospermo; y sus proporciones deseables están en función del uso final al que se destine el grano. La industria de las harinas instantáneas de maíz nixtamalizado requiere granos con $80 \%$ de endospermo, de modo que todos los maíces incluidos en este estudio cumplen con tal requerimiento. Las variedades de maíces criollos analizadas mostraron diferencias significativas en sus contenidos de endospermo, pericarpio y germen. El Cuadro 1 muestra que el maíz Pepitilla tuvo el valor más alto de pericarpio (7.55 \%). La industria de las harinas nixtamalizadas requiere maíces con un máximo de $5.5 \%$ de pericarpio, por lo que esta accesión no cumple con este requerimiento. Sin embargo los industriales de la masa y la tortilla no tienen establecido un valor para esta estructura, con la ventaja de que el pericarpio proporciona mayor cantidad de hidrocoloides convenientes para la textura de la tortilla (Vázquez et al., 2003), lo que corresponde con los resultados aquí encontrados (Cuadro 2).

La accesión utilizada en este estudio perteneciente a la raza Elotes Cónicos presentó el mayor porcentaje de germen (12.87\%), en tanto que la accesión de las razas Cónico Norteño y Bolita mostraron los menores valores (10.24\%); sin embargo no hubo diferencia significativa en el contenido de lípidos (Cuadro 1). La industria aceitera prefiere granos con la mayor cantidad de germen, ya que eso aumentaría sus rendimientos (Cruz-Requena et al., 2011). Por el contrario, en la industria del almidón un alto contenido de germen tiene un efecto negativo sobre el rendimiento de harina, debido a que tanto el pericarpio como el germen del grano se remueven; por tanto, para la industria del almidón los maíces con alto contenido de germen o de 


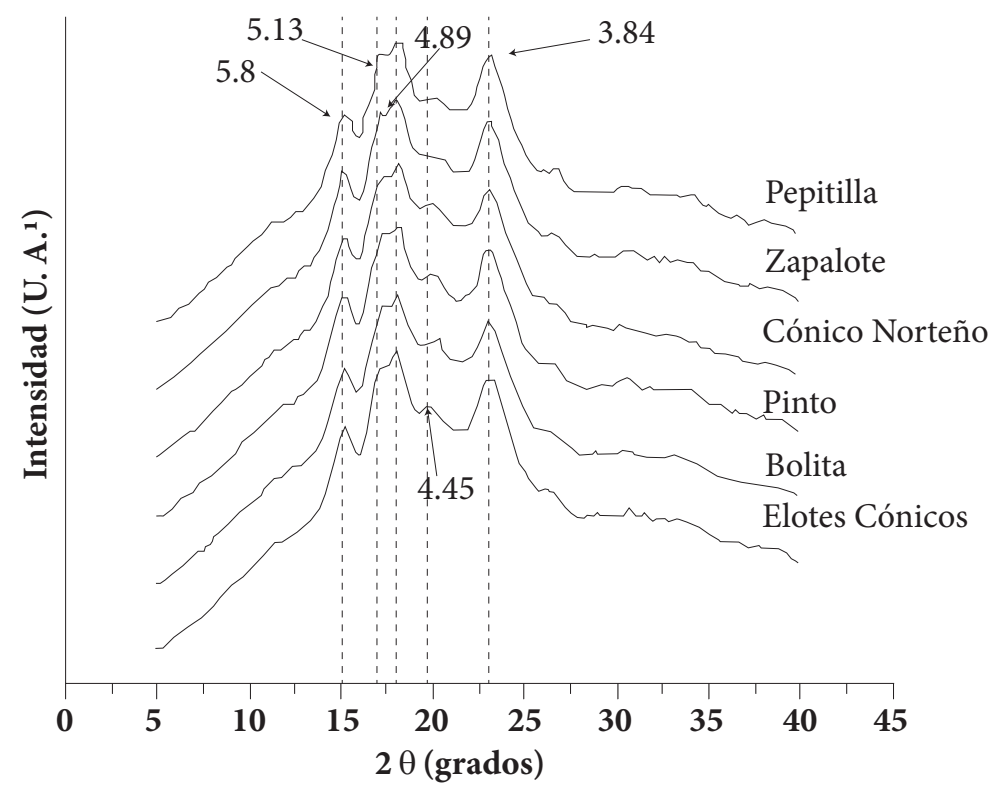

Figura 1. Difracción de rayos $\mathrm{X}$ de maíces nativos. ${ }^{1} \mathrm{U} . \mathrm{A}$. Unidades arbitrarias.

Cuadro 2. Medias de las propiedades térmicas y calidad nixtamalera de los maíces nativos.

\begin{tabular}{|c|c|c|c|c|c|c|c|c|c|c|}
\hline \multirow{3}{*}{ Maíz nativo } & \multicolumn{3}{|c|}{ Propiedades térmicas } & \multicolumn{7}{|c|}{ Calidad nixtamalera } \\
\hline & \multirow{2}{*}{$\begin{array}{c}\mathrm{VR}^{\xi} \\
\left(\mathrm{URV}^{1}\right)\end{array}$} & \multirow{2}{*}{$\begin{array}{c}\text { Entalpía } \\
(\mathrm{J} / \mathrm{g})\end{array}$} & \multirow[b]{2}{*}{$\operatorname{Tg}\left({ }^{\circ} \mathrm{C}\right)$} & \multicolumn{3}{|c|}{ Masa } & \multicolumn{4}{|c|}{ Tortilla } \\
\hline & & & & $\mathrm{RM}^{\mathrm{d}}$ & $\begin{array}{c}\text { Dureza } \\
(\mathrm{g}-\mathrm{F})\end{array}$ & $\begin{array}{c}\text { Adhesión } \\
\text { (g-F) }\end{array}$ & $\mathrm{RT}^{\&}$ & $\operatorname{PP}^{\xi \xi}(\%)$ & $\begin{array}{l}\text { Corte } \\
(\mathrm{g}-\mathrm{F})\end{array}$ & $\begin{array}{c}\text { Tensión } \\
\text { (g-F) }\end{array}$ \\
\hline Elotes cónicos & $301 \mathrm{a}$ & $7.07 \mathrm{a}$ & $72.39 \mathrm{~cd}$ & $2.10 \mathrm{~b}$ & $164 \mathrm{~b}$ & $14.45 \mathrm{a}$ & $1.69 \mathrm{a}$ & $19.49 \mathrm{bc}$ & $1340 \mathrm{c}$ & $290 \mathrm{ab}$ \\
\hline Bolita & $130 \mathrm{~b}$ & $6.37 \mathrm{a}$ & $72.91 \mathrm{c}$ & $2.01 \mathrm{~b}$ & $292 \mathrm{a}$ & $24.89 \mathrm{ab}$ & $1.60 \mathrm{~b}$ & $20.01 \mathrm{~b}$ & $3340 \mathrm{a}$ & $280 \mathrm{ab}$ \\
\hline Pinto & $302 \mathrm{a}$ & $6.87 \mathrm{a}$ & $72.14 \mathrm{~d}$ & $2.27 \mathrm{a}$ & $210 \mathrm{ab}$ & $15.14 \mathrm{a}$ & $1.59 \mathrm{~b}$ & $27.95 \mathrm{a}$ & $1450 \mathrm{c}$ & $160 \mathrm{c}$ \\
\hline $\mathrm{CN}^{\dagger}$ & $253 \mathrm{a}$ & $7.01 \mathrm{a}$ & $73.60 \mathrm{~b}$ & $2.02 \mathrm{~b}$ & $200 \mathrm{ab}$ & $27.53 \mathrm{ab}$ & $1.66 \mathrm{ab}$ & $17.58 \mathrm{c}$ & $1450 \mathrm{c}$ & $140 \mathrm{c}$ \\
\hline Zapalote & $262 \mathrm{a}$ & $7.65 \mathrm{a}$ & $72.49 \mathrm{~cd}$ & $2.07 \mathrm{~b}$ & $227 \mathrm{ab}$ & $33.99 \mathrm{~b}$ & $1.65 \mathrm{ab}$ & $20.10 \mathrm{~b}$ & $1983 \mathrm{~b}$ & $273 \mathrm{~b}$ \\
\hline Pepitilla & $253 \mathrm{a}$ & $8.55 \mathrm{a}$ & $74.84 \mathrm{a}$ & $2.03 \mathrm{~b}$ & $188 \mathrm{~b}$ & $38.54 \mathrm{~b}$ & $1.64 \mathrm{ab}$ & $18.84 \mathrm{bc}$ & $1960 \mathrm{~b}$ & $340 \mathrm{a}$ \\
\hline
\end{tabular}

Medias con letras iguales en cada columna no son estadísticamente diferentes (Tukey, 0.05). $\mathrm{CN}^{\dagger}=$ Cónico Norteño; ${ }^{\xi}$ Viscosidad relativa; ${ }^{1} \mathrm{RVU}=$ unidades relativas de viscosidad (1 URV = $12 \mathrm{cPa}$ ); ${ }^{\tau}$ Temperatura inicial; ${ }^{\top}$ Temperatura pico; ${ }^{\Psi}$ Temperatura final; ${ }^{\delta}$ Rendimiento de masa (kg de harina/kg de masa); ${ }^{\&}$ Rendimiento de tortilla (kg de tortilla/kg de masa); ${ }^{\xi} \mathrm{PP}=$ pérdida de peso.

pericarpio no son adecuados (Lin et al., 2002).

El estudio de la composición química del maíz es de suma importancia para establecer diferencias entre cultivares y para destinarlos a un uso final. El contenido de proteínas fluctuó desde 9.65 \% (Elotes Cónicos) hasta 11.93 \% (Pepitilla) (Cuadro 1). Vázquez et al. (2003) reportaron valores semejantes de proteína y lípidos para las accesiones de las razas Bolita, Pepitilla y Zapalote. El porcentaje de cenizas no varió en los maíces estudiados, excepto en la accesión de Pepitilla, la cual presentó el menor valor (1.06 \%). El contenido de cenizas no es usado como un indicador de calidad.

\section{Propiedades térmicas}

El análisis de varianza mostró diferencias significativas en la viscosidad relativa y temperatura de gelatinización de los maíces estudiados (Cuadro 2). La viscosidad relativa es el punto máximo del perfil de viscosidad e indica que la mayoría de los gránulos de almidón alcanzaron su máximo hinchamiento, después del cual se da el colapso; por tanto, la viscosidad relativa es un indicador de la capacidad de absorción de agua que tiene el almidón (Beta et al., 2001; Wang y Wang, 2002). 
No se detectaron diferencias significativas entre los maíces evaluados en cuanto a los valores de viscosidad relativa (Cuadro 2), excepto en la accesión de la raza Bolita (130 URV) que desarrolló la menor viscosidad y la mayor dureza (14.6 kg-F). Un comportamiento similar fue reportado por Narváez et al. (2007b) al investigar el efecto de la dureza del maíz sobre el perfil de viscosidad; estos autores encontraron que las razas que desarrollaron viscosidades altas fueron las de menor dureza, y asociaron el desarrollo de la viscosidad con el grado de compactación de los gránulos de almidón, ya que los maíces suaves presentan un menor grado de compactación y un mayor tamaño de gránulos de almidón, lo que facilita de alguna manera la difusión del agua dentro del grano y permite desarrollar una mayor viscosidad.

La entalpía de gelatinización no presentó variación entre los maíces estudiados (Cuadro 2). Esta característica térmica está asociada con la disociación de las dobles hélices de la amilopectina y con la fusión de los cristales del almidón (Chiotelli y Le Meste, 2002), y correlacionó con el porcentaje de cristalinidad $(\mathrm{r}=0.482, \mathrm{P}<0.05)$. La matriz de correlación indicó que la entalpía de gelatinización presentó una asociación significativa con el PCG $(r=-0.671$, $\mathrm{P}<0.05)$. Resultados semejantes fueron reportados previamente por Sasaki y Matsuki (1998).

Las temperaturas de gelatinización (Cuadro 2) presentaron diferencias significativas entre los maíces estudiados. Los valores fluctuaron entre $72.14^{\circ} \mathrm{C}$ del maíz de la raza Pinto y $74.84^{\circ} \mathrm{C}$ en la accesión de la raza Pepitilla. La diferencia en las temperaturas de gelatinización del almidón se debe principalmente a diferencias en el grado de cristalinidad de los mismos (Narváez-González et al., 2006; Narváez et al., 2007 b). Según Méndez et al. (2005), los maíces con mayor temperatura de gelatinización se pueden utilizar en la producción de harinas de maíz.

\section{Calidad nixtamalera de los maíces}

En el Cuadro 2 se muestran los valores del rendimiento, cohesión y adhesión de masa. El rendimiento de masa fue inversamente proporcional al tamaño del gránulo de almidón, TGA $(\mathrm{r}=-0.710, \mathrm{P}<0.05)$. El maíz de la raza Pinto presentó el mayor rendimiento de masa $(2.27 \mathrm{~kg}$ de masa/ $\mathrm{kg}$ de harina) y fue significativamente diferente de los otros maíces estudiados.

Los genotipos analizados mostraron diferencias significativas en sus valores de cohesión de la masa (Cuadro 2). La masa con menor fuerza de cohesión fue la obtenida del maíz de la raza Elotes Cónicos (164 kg-F), en tanto que el maíz de la raza Pinto fue el que presentó el mayor valor (210 kg-F). La fuerza a la adhesión de la masa también pre- sentó diferencias significativas entre los maíces estudiados, ya que las accesiones de Elotes Cónicos y Pinto resultaron estadísticamente inferiores $(\mathrm{P}<0.05)$ que las accesiones de Zapalote y Pepitilla. En las masas nixtamalizadas se requiere cierta fuerza de adhesividad para que el material se pueda troquelar (Ramírez y Ortega, 1994), ya que sin adhesividad carece de la consistencia necesaria para formar la tortilla, $y$ una masa demasiado adhesiva (pegajosa) tampoco lo permite (Arámbula et al., 2001).

En cuanto a las variables analizadas de calidad de las tortillas (rendimiento de tortilla, RT; fuerza al corte, FC; y la fuerza a la tensión, FT), el maíz de la raza Pinto fue el que presentó el mayor rendimiento de masa $(2.27 \mathrm{~kg}$ de masa/kg de harina); sin embargo, también presentó la mayor pérdida de peso (27.95\%), y por ende tuvo el menor rendimiento de tortilla ( $1.59 \mathrm{~kg}$ tortilla/ $\mathrm{kg}$ de masa), y produjo tortillas suaves y poco resistentes (valores bajos de FC y FT). Los rendimientos de masa y tortilla dependen de la capacidad del grano para absorber agua y retenerla durante las etapas del proceso; particularmente la tortilla pierde una cantidad importante de agua durante la formación de la "ampolla", que es la etapa final del cocimiento (Salinas y Aguilar, 2010).

Los análisis de textura consideran las fuerzas al corte y a la tensión, medidas que simulan la acción de los molares al masticar y el rompimiento de la tortilla con la mano, respectivamente; las tortillas deben ser suaves, pero a la vez deben tener la capacidad de resistir la humedad que se adiciona al momento de añadir algún guiso para hacer "tacos", es decir tortillas enrolladas que contienen el guiso (GaytánMartínez et al., 2012). La fuerza al corte presentó correlación significativa con el índice de flotación $(\mathrm{r}=-0.804, \mathrm{P}<$ $0.05)$, con viscosidad relativa $(\mathrm{r}=0.846, \mathrm{P}<0.05)$, con cristalinidad $(\mathrm{r}=-0.814, \mathrm{P}<0.05)$, contenido de germen $(\mathrm{r}=$ $-0.681, \mathrm{P}<0.05)$ y rendimiento de masa $(\mathrm{r}=0.780, \mathrm{P}<0.05)$. Los valores encontrados de fuerza al corte oscilaron entre 1450 g-F en las accesiones de Pinto y Cónico Norteño; y $3340 \mathrm{~g}$-F en la accesión de Bolita. La fuerza a la tensión (Cuadro 2) de las tortillas mostró diferencias significativas entre los maíces estudiados $(P<0.05)$, y el rango fue de 140 g-F en la accesión de Cónico Norteño a 340 g-F en la accesión de Pepitilla.

Un aspecto crítico en la textura de la tortilla es la homogeneidad y grado de hidratación de los granos de maíz, así como del grado de gelatinización del almidón (Bedolla y Rooney, 1984). En la industria de las harinas nixtamalizadas la textura de la tortilla no representa un problema, ya que esto se puede solucionar con la adición de gomas (Flores-Farías et al., 2000); sin embargo, los industriales de la masa y la tortilla afirman que se requieren maíces que por sí solos proporcionen una textura de tortilla apropiada, 
por lo que es importante el contenido de pericarpio porque es donde se encuentran gomas naturales que benefician la textura de la tortilla (Gaytán-Martínez et al., 2012).

\section{Selección de los maíces en relación a la calidad para su uso final}

En este aspecto también se detectó diferencia entre los maíces analizados. La agrupación de los maíces de la Figura 2 se basa en las variables físicas, químicas, térmicas y de calidad nixtamalera del grano. De la variabilidad total encontrada en las seis muestras de maíces, $82.6 \%$ se explicó con los 3 primeros componentes principales (CP); los dos primeros explicaron $65.8 \%$ de la variación. En el CP1 las variables sobresalientes están relacionadas con las propiedades físicas y térmicas (entalpía, índice de flotación, contenidos de endospermo y pericarpio, y grado de cristalinidad), en tanto que en el CP2 sobresalen las variables asociadas con la calidad nixtamalera, rendimiento de masa, fuerza de adhesión, pérdida de peso y fuerza al corte.

Para realizar la clasificación del uso final de los maíces analizados (Cuadro 3) se consideraron cuatro usos principales: industria de las harinas nixtamalizadas (IHN), industria de la masa y la tortilla (IMyT), industria de las botanas (B), e industria de almidones o molienda seca (A). La designación del uso final de los maíces evaluados se hizo de acuerdo con la metodología propuesta por Figueroa (1985) y con las modificaciones hechas por Mauricio et al. (2004). Además se consideraron los parámetros de calidad establecidos en la NMX-FF-034-1995-SCFI y otros reportes de diversos investigadores (Salinas y Aguilar, 2010; Vásquez et al., 2003; Andrio et al., 2011).

Según Salinas y Aguilar (2010), en el país existen dos tipos de industrias que procesan el grano de maíz mediante la nixtamalización: la IMyT y la IHN. Sin embargo, en la literatura se hace una diferenciación entre las IHN, las que van dirigidas a la producción de tortillas y las que se destinan a la producción de botanas; la diferencia estriba en que la primera requiere que los granos de maíz tengan un mayor rendimiento de tortilla, es decir, que formen un gel que evite la pérdida de agua durante la cocción, mientras que en la de botanas requieren una alta pérdida de peso durante esta etapa, lo que se traducirá en productos más crujientes y con menor absorción de grasa (Andrio et al., 2011). De acuerdo con lo anterior, el maíz Bolita presenta un alto potencial de uso en la industria de botanas nixtamalizadas.

Conforme a los requerimientos de calidad para la IHN destinadas a elaborar tortilla descritos en la Norma Oficial Mexicana para maíz nixtamalizado (NMX-FF-034-1995SCFI), el maíz Cónico Norteño cumple con las especificaciones de esta industria.

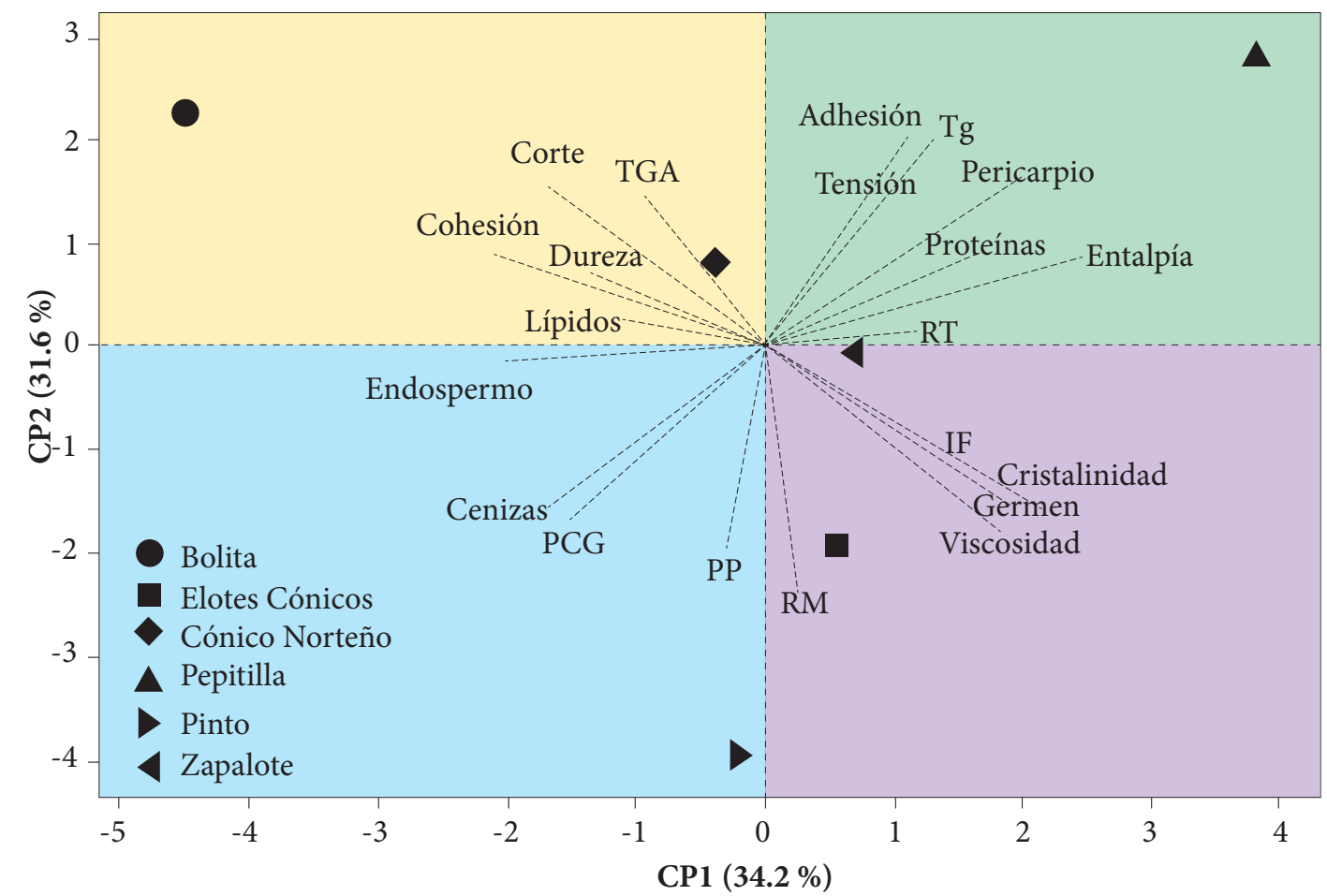

Figura 2. Dispersión de maíz nativos estudiados con base en los dos primeros componentes principales (CP1 y CP2). 
Cuadro 3. Parámetros de calidad de maíces para la industria.

\begin{tabular}{|c|c|c|c|c|}
\hline \multirow{2}{*}{ Variable } & \multicolumn{4}{|c|}{ Industria } \\
\hline & $\mathrm{IHN}^{\mathrm{x}}$ & $\mathrm{IMyT}^{\dagger}$ & $\mathrm{IB}^{+\dagger}$ & IEA $^{9}$ \\
\hline Peso de cien granos & $\leq 20$ & $\leq 18$ & $\leq 27.7 \mathrm{~g}$ & $\leq 32 \mathrm{~g}$ \\
\hline Índice de flotación & $>20 \%$ & $>40 \%$ & $>12 \%$ & $\leq 50 \%$ \\
\hline Dureza & $>12 \mathrm{~kg}-\mathrm{F}$ & $>11 \mathrm{~kg}-\mathrm{F}$ & $\leq 13 \mathrm{~kg}-\mathrm{F}$ & $>10 \mathrm{~kg}-\mathrm{F}$ \\
\hline Endospermo & $\leq 78 \%$ & $\leq 78 \%$ & $\leq 78 \%$ & $\leq 850 \%$ \\
\hline Pericarpio & $>5 \%$ & $>5 \%$ & $>5 \%$ & $>5 \%$ \\
\hline Germen & $>10 \%$ & $>10 \%$ & $>13 \%$ & $>10 \%$ \\
\hline Viscosidad relativa & $\leq 220 \mathrm{URV}$ & $\leq 220 \mathrm{URV}$ & $\leq 180 \mathrm{URV}$ & $\leq 320 \mathrm{URV}$ \\
\hline Pérdida de peso tortilla & $>16 \%$ & $>16 \%$ & $\leq 30 \%$ & $\leq 20 \%$ \\
\hline
\end{tabular}

En el caso de la IMyT, los valores de los parámetros deseable no son muy claros; sin embargo, se infiere que requiere granos de dureza intermedia, con alta capacidad de absorción de agua y, por consiguiente, con altos rendimientos de masa y tortilla, así como tortillas con baja resistencia al corte y a la tensión (Narváez-González et al., 2006; Narváez et al., 2007a; Vásquez et al., 2003; Salinas y Aguilar, 2010; Andrio et al., 2011). Las accesiones de las razas Cónico Norteño, Zapolote y Pepitilla presentaron características apropiadas para esta industria.

En contraparte, la industria refinadora de almidón prefiere granos suaves o de baja densidad porque tienen menor tiempo de remojo en la solución de dióxido de azufre y presentan mayor contenido de almidón y menor contenido de proteína, comparados con los granos de textura vítrea, así como alto peso de 100 granos (Serna, 1999). Por ello, para esta industria los maíces deben tener altos valores de viscosidad, alto porcentaje de endospermo y bajos porcentajes de pericarpio y germen. Los maíces analizados que pueden ser destinados a este fin son los de las razas Pinto y Elotes Cónicos.

\section{CONCLUSIONES}

Los resultados obtenidos muestran que el uso final se determina principalmente con las características físicas y químicas del grano, posteriormente por sus propiedades térmicas, y por ultimo por las características de calidad de masa y tortilla. Los maíces analizados de las razas Cónico Norteño, Zapalote y Pepitilla presentaron características apropiadas para la industria de la masa y la tortilla; la accesión de Cónico Norteño es apropiada también para la industria de las harinas nixtamalizadas; la accesión de Bolita presenta un mejor potencial para ser usada en la industria de botanas nixtamalizadas; los maíces de las razas Pinto y Elotes Cónicos son más apropiados para la industria de al- midones. Es importante resaltar que como resultado de este estudio, que aunque solo incluyó una accesión por raza y que la variabilidad genética intrarracial puede ser muy alta, los maíces criollos pueden ser orientados a un uso específico más apropiado, a fin de que el productor obtenga una transacción económica favorable al dirigirse al nicho de mercado apropiado donde puede obtener un sobreprecio por las características de su grano.

\section{AGRADECIMIENTOS}

A "MASAGRO: Modernización sustentable de la agricultura tradicional", un programa de SAGARPA-CIMMYT, por su apoyo financiero. A la revisión técnica y editorial de M. Willcox y N. Palacios Rojas. Al M. C. José J. Velés Medina y al Q. en A. Martín Adelaido Hernández Landaverde, del Cinvestav-Querétaro, por su apoyo técnico.

\section{BIBLIOGRAFÍA}

AOAC, Association of Official Analytical Chemists (2002) Official Method: Protein 954, Oil 920.39 and 923.03Ash Official Methods of Analysis (17th ed). Association of Official Analytical Chemists. Washington, DC. USA.

Andrio E E, S Rodríguez, A Palomo, A Espinosa, F Cervantes, E Gutiérrez, G Arámbula, M Mendoza (2011) Caracterización del potencial agronómico de maíces criollos para el mejoramiento genético. In: Amplitud, Mejoramiento, Usos y Riesgos de la Diversidad Genética de Maíz en México. R E Preciado O, S Montes H (eds). Sociedad Mexicana de Fitogenética A. C. pp:70-71.

Aragón C F, S Taba, J M Hernández C, J D Figueroa C, V Serrano A, F H Castro (2006) Actualización de la Información sobre los Maíces Criollos de Oaxaca. Instituto Nacional de Investigaciones Forestales Agrícolas y Pecuarias, Libro Técnico No. 6. 345 p.

Arámbula V G, A L Barrón, H J González, M E Moreno, B G Luna (2001) Efecto del tiempo de cocimiento y reposo del grano de maíz (Zea mays L.) nixtamalizado, sobre las características fisicoquímicas, reológicas, estructurales y texturales del grano, masa y tortillas de maíz. Arch. Latinoam. Nutr. 51:187-194.

Bedolla S, L W Rooney (1984) Characteristic of U.S. Mexican instant maize flour for tortilla and snack preparation. Cereal F. World 29:732-735.

Beta T, H Corke, J R N Taylor, L W Rooney (2001) Effect of steeping treatment on pasting and thermal properties of sorghum 
starches. Cereal Chem. 78:303-306.

Bocharmikova I, L A Wasserman, A V Krivandin, J Fornal, W B Blaszczak, V Y Chernykh, A Schiraldi, V P Yuryev (2003) Structure and thermodynamic melting parameters of wheat starches with different amylose content. J. Thermal Anal. Calorim. 74:681-69.

Cruz-Requena M, R Rodríguez-Herrera, C N Aguilar-González, J Espinoza-Velazquez, M Gaytán-Martínez, J D FigueroaCárdenas (2011) Alkaline cooking quality of polyembryonic and non-polyembryonic maize populations. Adv. J. Food Sci. Technol. 3:259-268.

Chiotelli E, M Le Meste (2002) Effect of small and large wheat starch granules on thermomechanical behavior of starch. Cereal Chem. 779:286-293.

Figueroa C J D (1985) Métodos para Evaluar la Calidad Maltera de la Cebada. Secretaría de Agricultura y Recursos Hidráulicos (ed). Tema Didáctico Núm. 17. México, D. F. pp:30-67

Flores-Farías R, F Martínez-Bustos, Y Salinas-Moreno, J K Chang, J González-Hernández, E Ríos (2000) Physicochemical and rheological characteristics of commercial nixtamalised Mexican maize flours for tortillas. J. Sci. Food Agric. 80:657-664.

Gaytán-Martínez M, J D Figueroa C, P A Vázquez-Landaverde, E Morales-Sánchez, H E Martínez-Flores, M L Reyes-Vega (2012) Caracterización fisicoquímica, funcional y química de harinas nixtamalizadas de maíz obtenidas por calentamiento óhmico y proceso tradicional. CyTA J. Food 10:182-195.

Lin Y P, A Aboubacar, B E Zehr, B R Hamaker (2002) Corn dry-milled grit and flour fractions exhibit differences in amylopectin fine structure and gel texture. Cereal Chem. 79:354-358.

Mauricio S R A, J D Figueroa C, S Taba, M L Reyes V, F Rincón S, A Mendoza G (2004) Caracterización de accesiones de maíz por calidad de grano y tortilla. Rev. Fitotec. Mex. 27:213-222.

Méndez M G, J Solorza F, V M Velázquez, N Gómez M, O Paredes L, L A Bello P (2005) Composición química y caracterización calorimétrica de híbridos y variedades de maíz cultivadas en México. Agrociencia 39:267-274.

Narváez G E D, J D Figueroa C, S Taba (2007a) Aspectos microestructurales y posibles usos del maíz de acuerdo a su origen geográfico. Rev. Fitotec. Mex. 30:321-325.

Narváez G E D, J D Figueroa C, S Taba, E Castaño T, R A Martínez P (2007 b) Efecto del tamaño del gránulo de almidón en maíz en sus propiedades térmicas y de pastificado. Rev. Fitotec. Mex. 30:269-277.

Narváez-González E D, J D Figueroa-Cárdenas, S Taba, F Rincón S (2006) Kernel microstructure of Latin American races of maize and their thermal and rheological properties. Cereal Chem. 83:605-610.

NMX-FF-034-1995-SCFI, Secretaría de Economía (1995) Productos alimenticios no industrializados para consumo humano - cerea- les: maíz blanco para proceso alcalino para tortillas de maíz y productos de maíz nixtamalizado - especificaciones y métodos de prueba. Disponible en: http://200.77.231.100/work/normas/ nmx/1995/nmx-ff-034-1995.pdf (Noviembre 2009).

Patindol J, Y J Wang (2003) Fine structures and physicochemical properties of starches from chalky and translucent rice kernels. J. Agric. Food Chem. 51:2777-2784.

Ramírez W B, F Ortega (1994) Evaluaciones de las propiedades reológicas y texturales de masa y tortillas de maíces comerciales. Tecnol. Alim. 2:5-8.

Salinas M Y, L Aguilar M (2010) Efecto de la dureza del grano de maíz (Zea mays L.) sobre el rendimiento y calidad de la tortilla. Ing. Agríc. Biosist. 2:5-11.

Salinas M Y, G Vázquez C (2006) Metodologías de Análisis de la Calidad Nixtamalera-Tortillera en Maíz. Folleto Técnico No. 23. INIFAP-Campo Experimental Valle de México. México. 91 p.

Salinas M Y, J L Arellano V, F Martínez B (1992) Propiedades físicas, químicas y correlaciones de maíces híbridos precoces para Valles Altos. Arch. Latinoam. Nutr 42:161-167.

SAS Institute (2004) SAS/STAT ${ }^{8} 9.1$ User's Guide. Cary, NC: SAS Institute Inc. USA. $5121 \mathrm{p}$.

Sasaki T, J Matsuki (1998) Effect of wheat starch structure on swelling power. Cereal Chem. 75:525-529.

Serna S S O (1999) Utilización industrial del maíz en México. In: Memorias del 2do. Taller Nacional de Especialidades de Maíz. J Espinoza V, C J del Bosque (eds). Saltillo, Coah. pp:77-105.

Trujillo C A (2003) Guía para Seleccionar Semillas de Maíz Criollo y Variedades de Polinización Libre. Folleto Técnico No. 49. INIFAP-Campo Zacatepec, Morelos. México. $17 \mathrm{p}$.

United States Grains Council (2001) The 2000-2001 value-enhanced grains quality report. Washington, DC: vailable on the World Wide Web: http://www.vegrains.org. (Diciembre 2010).

Vázquez C M G, L Guzmán B, J L Andrés G, F Márquez S, J Castillo M (2003) Calidad de grano y tortilla de maíces criollos y sus retrocruzas. Rev. Fitotec. Mex. 26:231-238.

Wang S, L Hongyan, G Wenyuan, Ch Haixia, Y Jiugao, X Peigen (2006) Characterization of new starches separated from different Chinese yam (Dioscorea opposita Thunb.) cultiva rs. Food Chem. 99:30-37.

Wang Y, L Wang (2002) Structures of four waxy rice starches in relation to thermal, pasting, and textural properties. Cereal Chem.79:252 256.

Wellhausen E J, L M Roberts, E Hernández, P C Mangelsdorf (1952) Races of Maize in Mexico: Their Origin, Characteristics and Distribution. 1st ed. The Bussey Institution of Harvard University. 223 p.

Zobel H F (1988) Starch crystal transformations and their industrial importance. Starch 40:1-7. 